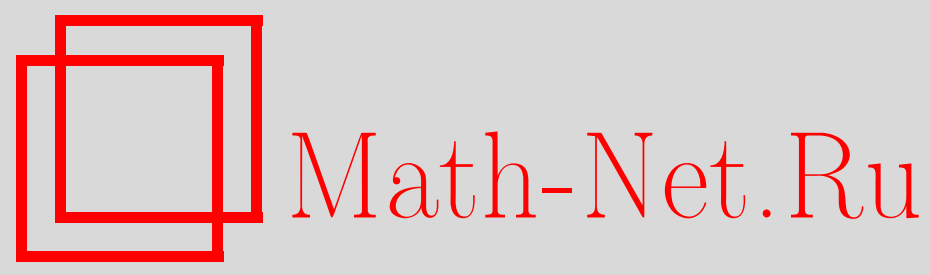

И. Р. Каюмов, О гипотезе Бреннана для специального класса функций, Матем. заметки, 2005, том 78, выпуск 4, 537541

DOI: https://doi.org/10.4213/mzm2612

Использование Общероссийского математического портала Math-Net.Ru подразумевает, что вы прочитали и согласны с пользовательским соглашением http://www. mathnet.ru/rus/agreement

Параметры загрузки:

IP: 54.198 .187 .58

26 апреля 2023 г., 17:22:39

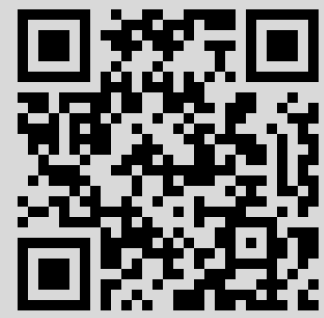




\section{О ГИПОТЕЗЕ БРЕННАНА ДЛЯ СПЕЦИАЛЬНОГО КЛАССА ФУНКЦИЙ}

\section{И.Р. Каюмов}

В работе доказьвается гипотеза Бреннана для конформных отображений $f$ круга $\{z:|z|<1\}$ в предположении, что у функции $\log \left(z f^{\prime}(z) / f(z)\right)$ коэффициенты Тейлора в нуле неотрицательны. Кроме того, получены неравенства для интегральных средних по окружности $|z|=r$ квадрата модуля функции $z f^{\prime}(z) / f(z)$.

Библиографияя: 8 названий.

Пусть $\Omega$ - односвязная область на плоскости, граница которой состоит из более, чем одной точки, и $\varphi-$ конформное отображение области $\Omega$ на круг $\mathbb{D}=\{z:|z|<1\}$. Бреннаном [1] была высказана гипотеза о том, что $\varphi^{\prime} \in L_{p}(\Omega)$ при $4 / 3<p<4$, т.е.

$$
\int_{\Omega}\left|\varphi^{\prime}\right|^{p} \mathrm{~d} x \mathrm{~d} y<\infty, \quad \frac{4}{3}<p<4 .
$$

Если $\Omega$ - плоскость с разрезом по некоторому лучу, то при $p \notin(4 / 3,4)$

$$
\int_{\Omega}\left|\varphi^{\prime}\right|^{p} \mathrm{~d} x \mathrm{~d} y=\infty .
$$

Пусть $f: \mathbb{D} \rightarrow \Omega$ - конформное отображение единичного круга $\mathbb{D}$ на $\Omega$. Из результатов, полученных в работе [2], следует, что гипотеза Бреннана эквивалентна соотношению $\beta_{f}(-2) \leqslant 1$, где

$$
\beta_{f}(t)=\limsup _{r \rightarrow 1} \frac{\log \int_{0}^{2 \pi}\left|f^{\prime}\left(r e^{i \theta}\right)\right|^{t} \mathrm{~d} \theta}{|\log (1-r)|}
$$

- спектр интегральных средних.

Для достаточно больших отрицательных $t$, как это установлено Карлесоном и Макаровьц $[3], \beta_{f}(t) \leqslant|t|-1$. Равенство достигается для функции Кёбе $f(z)=z /(1-z)^{2}$. Там же показано, что для интервала $(-2,1 / 3]$ спектр интегральных средних максимизируется функцией, отображающей круг $\mathbb{D}$ на область с фрактальной границей. Гипотеза Бреннана эквивалентна тому, что точка $t=-2$ является точкой фазового перехода для функции $\sup _{f} \beta_{f}(t):$ при $t \leqslant-2$ эта функция должна быть линейна (если справедлива эта гипотеза), а при $-2<t \leqslant 1 / 3$ строго вьпукла. Здесь супремум берется по всем однолистньм аналитическим в круге $\mathbb{D}$ функциям. Отсюда видно, что сложность этой

Работа выполнена при частичной поддержке Российского фонда фундаментальных исследований, гранты № 05-01-00523 и № 03-01-00015, и фонда НИОКР АН РТ. 
проблемы связана с граничным поведением конформных отображений круга на области с фрактальными границами. Поскольку гипотезу Бреннана не удалось доказать прямыми методами геометрической теории функций, то эту гипотезу стали доказьвать для различных частных случаев. Бертильсон в своей диссертации [4] исследовал локальную версию гипотезы Бреннана для функций, близких к функции Кёбе. Бараньски, Вольберг и Здуник [5] доказали гипотезу Бреннана для всех односвязных областей притяжения бесконечности квадратичных полиномов. Другой важньй класс фракталов состоит из конформных отображений $f$, для которьх $\log f^{\prime}$ представим в виде лакунарного ряда Адамара, т.е.

$$
\log f^{\prime}=\sum_{k=0}^{\infty} a_{k} z^{n_{k}}, \quad \frac{n_{k+1}}{n_{k}} \geqslant q>1
$$

В нашей работе [6] доказано, что гипотеза Бреннана для таких функций верна, если $q \geqslant 15$.

В этой статьемы докажем гипотезу Бреннана в предположении, что тейлоровские коэффициенты функции $\log \left(z f^{\prime} / f\right)$ неотрицательны. А именно, мы показьваем, что справедлива

ТЕОРема. Предположим, что аналитическая функиия $f$ однолистна в круге $\mathbb{D}$, $f(0)=0$. Если тейлоровские коэффичиенты функиии $\log \left(z f^{\prime} / f\right)$ неотричательны, то $\beta_{f}(-2) \leqslant 1$, что әквивалентно достоверности гипотезы Бреннана в рассматриваемом случае. Равенство $\beta_{f}(-2)=1$ достигается, например, для функиии Kёбе $f=z /(1-z)^{2}$.

ДоКАЗАТЕЛЬСТВо. Без ограничения общности можно считать, что $f^{\prime}(0)=1$. В силу условия теоремы справедливо следующее равенство:

$$
\log \frac{z f^{\prime}(z)}{f(z)}=\sum_{k=1}^{\infty} a_{k} z
$$

где $a_{k} \geqslant 0$ при $k \geqslant 1$.

Пусть $b_{k}$ - тейлоровские коэффициенты функции $f /\left(z f^{\prime}\right), c_{k}-$ тейлоровские коэффициенты функции $z f^{\prime} / f$. В силу положительности $a_{k}$ и равенства

$$
\frac{f(z)}{z f^{\prime}(z)}=\exp \left(-\sum_{k=1}^{\infty} a_{k} z\right)
$$

ясно, что $\left|b_{k}\right| \leqslant c_{k}$

Отсюда при помощи равенства Парсеваля получаем неравенство

$$
\frac{1}{2 \pi} \int_{0}^{2 \pi}\left|\frac{f\left(r e^{i \theta}\right)}{r f^{\prime}\left(r e^{i \theta}\right)}\right|^{2} \mathrm{~d} \theta \leqslant \frac{1}{2 \pi} \int_{0}^{2 \pi}\left|\frac{r f^{\prime}\left(r e^{i \theta}\right)}{f\left(r e^{i \theta}\right)}\right|^{2} \mathrm{~d} \theta .
$$

Далее, силу равенства Парсеваля

$$
\frac{1}{2 \pi} \int_{0}^{2 \pi}\left|\frac{r f^{\prime}\left(r e^{i \theta}\right)}{f\left(r e^{i \theta}\right)}\right|^{2} \mathrm{~d} \theta=\sum_{k=0}^{\infty} c_{k}^{2} r^{2 k}=1+\sum_{k=1}^{\infty} k^{2}\left|\gamma_{k}\right|^{2} r^{2 k}=1+\sum_{k=1}^{\infty} k r^{k} k\left|\gamma_{k}\right|^{2} r^{k}
$$


где $\gamma_{k}$ - тейлоровские коэффициенты функции $\log (f(z) / z)$.

Милиным [7, с. 87] показано, что

$$
\sum_{k=1}^{\infty} k\left|\gamma_{k}\right|^{2} r^{2 k} \leqslant 2 \log M\left(r, \frac{f}{r}\right),
$$

где

$$
M\left(r, \frac{f}{r}\right)=\max _{|z|=r} \frac{|f(z)|}{r} .
$$

По известной теореме искажения $M(r, f / r) \leqslant 1 /(1-r)^{2}$. Неравенство (2) перепишется следующим образом:

$$
\sum_{k=1}^{\infty} k\left|\gamma_{k}\right|^{2} r^{2 k} \leqslant 4|\log (1-r)| .
$$

С другой стороны, ясно, что $c_{k}=k \gamma_{k}$ при $k \geqslant 1, c_{0}=1$.

Хорошо известно, что $k r^{k} \leqslant e^{-1} /(1-r)$. Поэтому,

$$
\frac{1}{2 \pi} \int_{0}^{2 \pi}\left|\frac{r f^{\prime}\left(r e^{i \theta}\right)}{f\left(r e^{i \theta}\right)}\right|^{2} \mathrm{~d} \theta \leqslant 1+\frac{e^{-1}}{1-r} \sum_{k=1}^{\infty} k\left|\gamma_{k}\right|^{2} r^{k}
$$

Таким образом, из неравенств (1) и (3) следует, что

$$
\frac{1}{2 \pi} \int_{0}^{2 \pi}\left|\frac{f\left(r e^{i \theta}\right)}{r f^{\prime}\left(r e^{i \theta}\right)}\right|^{2} \mathrm{~d} \theta \leqslant 1+\frac{4}{e} \frac{|\log (1-\sqrt{r})|}{1-r} .
$$

Комбинируя это неравенство с теоремой Кёбе об $1 / 4$, согласно которой $\left|f\left(r e^{i \theta}\right)\right| \geqslant r / 4$, окончательно получаем

$$
\frac{1}{2 \pi} \int_{0}^{2 \pi} \frac{1}{\left|f^{\prime}\left(r e^{i \theta}\right)\right|^{2}} \mathrm{~d} \theta \leqslant \frac{16}{2 \pi} \int_{0}^{2 \pi}\left|\frac{f\left(r e^{i \theta}\right)}{r f^{\prime}\left(r e^{i \theta}\right)}\right|^{2} \mathrm{~d} \theta \leqslant 16+\frac{64}{e} \frac{|\log (1-\sqrt{r})|}{1-r},
$$

откуда заключаем, что $\beta_{f}(-2) \leqslant 1$. Теорема доказана.

В качестве замечания отметим, что, несколько изменяя доказательство, нетрудно показать справедливость гипотезы Бреннана для функций, имеющих следующеепредставление:

$$
\log \frac{z f^{\prime}(z)}{f(z)}=\sum_{k=1}^{\infty} a_{k} z+g(z)
$$

где $a_{k} \geqslant 0$ при $k \geqslant 1$, а функция $g(z)$ удовлетворяет в круге $\mathbb{D}$ условию

$$
\operatorname{Re} g(z)=o(\log (1-|z|)) \quad \text { при }|z| \rightarrow 0 .
$$

При доказательстве теоремы был установлен следующий факт: для произвольной аналитической и однолистной в круге $\mathbb{D}$ функции $f(f(0)=0)$ справедливо неравенство

$$
\frac{1}{2 \pi} \int_{0}^{2 \pi}\left|\frac{r f^{\prime}\left(r e^{i \theta}\right)}{f\left(r e^{i \theta}\right)}\right|^{2} \mathrm{~d} \theta \leqslant 1+\frac{4}{e} \frac{|\log (1-\sqrt{r})|}{1-r} .
$$

Возникает естественньй вопрос: нельзя ли заменить “логарифмический” множитель в этом неравенстве некоторой абсолютной постоянной? Автору неизвестен ответ на этот вопрос в общем случае. Для некоторых случаев можно дать положительньй ответ на этот вопрос. Например, справедливо 
УТВЕРЖДЕНИЕ. Предположим, что аналитическая функция $f$ однолистна в круге $D, f(0)=f^{\prime}(0)-1=0$. Если

$$
\varlimsup_{r \rightarrow 1} M(r, f)(1-r)^{2}=\alpha>0,
$$

mo

$$
\frac{1}{2 \pi} \int_{0}^{2 \pi}\left|\frac{r f^{\prime}\left(r e^{i \theta}\right)}{f\left(r e^{i \theta}\right)}\right|^{2} \mathrm{~d} \theta \leqslant \frac{2 e^{-1} \log \left(\alpha^{-1}\right)}{1-r}+\frac{4 r \sqrt{2 \log \left(\alpha^{-1}\right)}}{1-r^{2}}+\frac{1+3 r^{2}}{1-r^{2}}
$$

əде

$$
M(r, f)=\max _{|z|=r}|f(z)| .
$$

При $\alpha=1$ равенство достигается для функции Кёбе $f(z)=z /(1-z)^{2}$.

ДокАЗАТЕЛЬСтво. Базилевичем (см. [8], [7, с. 82]) доказано неравенство

$$
\sum_{k=1}^{\infty} k\left|\gamma_{k}-\frac{2}{k} t_{0}^{k}\right|^{2} \leqslant 2 \log \frac{1}{\alpha}
$$

где $t_{0}$ - некоторое число на окружности $|z|=1, \gamma_{k}$ - тейлоровские коэффициенты функции $\log (f(z) / z)$. Через $c_{k}$ обозначим тейлоровские коэффициенты функции $z f^{\prime} / f$. Мы имеем

$$
\begin{aligned}
\frac{1}{2 \pi} \int_{0}^{2 \pi}\left|\frac{r f^{\prime}\left(r e^{i \theta}\right)}{f\left(r e^{i \theta}\right)}\right|^{2} \mathrm{~d} \theta= & \sum_{k=0}^{\infty}\left|c_{k}\right|^{2} r^{2 k}=1+\sum_{k=1}^{\infty} k^{2}\left|\gamma_{k}\right|^{2} r^{2 k} \\
= & 1+\sum_{k=1}^{\infty} k^{2}\left|\gamma_{k}-\frac{2}{k} t_{0}^{k}+\frac{2}{k} t_{0}^{k}\right|^{2} r^{2 k} \\
\leqslant & 1+\sum_{k=1}^{\infty} k^{2}\left|\gamma_{k}-\frac{2}{k} t_{0}^{k}\right|^{2} r^{2 k}+\sum_{k=1}^{\infty} k^{2}\left|\frac{2}{k} t_{0}^{k}\right|^{2} r^{2 k} \\
& +2 \sum_{k=1}^{\infty} k^{2}\left|\frac{2}{k} t_{0}^{k}\right|\left|\gamma_{k}-\frac{2}{k} t_{0}^{k}\right| r^{2 k} \\
= & 1+\sum_{k=1}^{\infty} k^{2}\left|\gamma_{k}-\frac{2}{k} t_{0}^{k}\right|^{2} r^{2 k}+4 \sum_{k=1}^{\infty} k\left|\gamma_{k}-\frac{2}{k} t_{0}^{k}\right| r^{2 k}+\frac{4 r^{2}}{1-r^{2}}
\end{aligned}
$$

Оценим суммы рядов в последнем выражении. Имеем

$$
\sum_{k=1}^{\infty} k^{2}\left|\gamma_{k}-\frac{2}{k} t_{0}^{k}\right|^{2} r^{2 k} \leqslant \frac{e^{-1}}{1-r} \sum_{k=1}^{\infty} k\left|\gamma_{k}-\frac{2}{k} t_{0}^{k}\right|^{2} r^{k} .
$$

Здесь мы использовали, как и при доказательстве теоремы, тот факт, что

$$
k r^{k}<\frac{e^{-1}}{1-r}
$$


Применяя неравенство Гёльдера, оценим другой ряд:

$$
\begin{aligned}
\sum_{k=1}^{\infty} k\left|\gamma_{k}-\frac{2}{k} t_{0}^{k}\right| r^{2 k} & \leqslant \sqrt{\sum_{k=1}^{\infty} k\left|\gamma_{k}-\frac{2}{k} t_{0}^{k}\right| r^{2 k} \sqrt{\sum_{k=1}^{\infty} k r^{2 k}}} \\
& =\frac{r}{1-r^{2}} \sqrt{\sum_{k=1}^{\infty} k\left|\gamma_{k}-\frac{2}{k} t_{0}^{k}\right| r^{2 k}}
\end{aligned}
$$

Теперь, используя неравенство (4), заключаем, что

$$
\frac{1}{2 \pi} \int_{0}^{2 \pi}\left|\frac{r f^{\prime}\left(r e^{i \theta}\right)}{f\left(r e^{i \theta}\right)}\right|^{2} \mathrm{~d} \theta \leqslant 1+\frac{2 e^{-1} \log \left(\alpha^{-1}\right)}{1-r}+\frac{4 r \sqrt{2 \log \left(\alpha^{-1}\right)}}{1-r^{2}}+\frac{4 r^{2}}{1-r^{2}}
$$

Утверждение доказано.

Автор благодарен Ф.Г. Авхадиеву за полезное обсуждение результатов статьи.

\section{СПИСОК ЦИТИРОВАННОЙ ЛИТЕРАТУРЫ}

[1] Brennan J. E. On the integrability of the derivative in conformal mapping // J. London Math. Soc. (2). 1978. V. 18. P. 261-272.

[2] Pommerenke Ch. On the integral means of the derivative of a univalent function // J. London Math. Soc. (2). 1985. V. 32. P. 254-258.

[3] Carleson L., Makarov N. G. Some results connected with Brennan's conjecture // Ark. Mat. 1994. V. 32. P. 33-62.

[4] Bertilsson D. On Brennan's conjecture in conformal mapping. Doctoral Thesis. Stockholm: Royal Inst. of Tech., 1999.

[5] Barański K., Volberg A., Zdunik A. Brennan's conjecture and the Mandelbrot set // Intern. Math. Res. Notices. 1998. V. 12. P. 589-600.

[6] Kayumov I. R. Integral means and the law of the iterated logarithm // Preprint № 8. Sweden: Institut Mittag-Leffler, 2002.

[7] Милин И. М. Однолистные функции и ортонормированные системы. М.: Наука, 1971.

[8] Базилевич И.Е. Об одном критерии однолистности регулярных функций и дисперсии их коэффициентов // Матем. сб. 1967. Т. 74. № 1. С. 133-146.

Научно-исследовательский институт

Поступило

математики и механики им. Н. Г. Чеботарёва

17.12 .2004

Казанского государственного университета

E-mail: ikayumov@mail.ru

Исправленный вариант

11.04.2005 